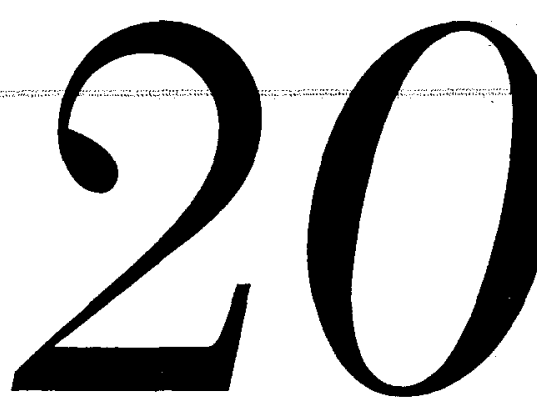

\title{
Commentary Goals and the Intentions Meant to Fulfill Them
}

\author{
PETER M. GOLLWITZER \\ Department of Psychology \\ New York University and University of Konstanz \\ ANNA-LISA COHEN \\ Department of Psychology \\ New York University
}

\begin{abstract}
$A$ $\mathrm{s}$ described in the first paragraph by Dismukes (chap. 19, this volume), a forgotten intention had drastic and tragic consequences for Flight 1141. Without a doubt, all of the crew members, engineers, pilots, and air traffic controllers shared a common goal: to make sure that the plane flew safely, ensuring the health and welfare of all passengers. However, one intention in a series of intentions meant to fulfill that goal, namely to prepare the plane for takeoff, was forgotten.

Typically in the prospective memory literature, the term goal is used very seldom and it is memory for intention that is the focus of scholarly inquiry. However, it may be useful to consider the relative obscurity of the term goal in the prospective memory literature as it may reflect that we are missing one piece of the puzzle. Baldwin (1897; as cited in Olson, Astington \& Zelazo, 1999) defined intentional action in terms of goal-directed behavior. He described it as "the emergence of desire, deliberation, and effort: the conscious representation of a goal, the active consideration of alternative means and ends, and the feeling accompanying the selection and execution of a plan (p. 2, Olson et al., 1999). The term goal is used to refer to the idea that a mental representation of a desired future state is formed and in turn directs behavior to find a way to achieve that state. It may be that the airline employees in
\end{abstract}


the Dismukes example had no problem maintaining their goal in mind, but their failure to implement their various intentions in an effort to achieve the goal led to trouble. Substantial failures of prospective memory may thus occur not merely due. to cognitive failure, but also due to implemental problems.

In fact, Wilson and Park (chap. 18, this volume) propose that aspects of prospective memory related to medication adherence are very sensitive to contextual (as opposed to cognitive) factors. They present the counterintuitive result that younger adults are more at risk than older adults for making medication adherence errors. Wilsoh and Park found that those who were most at risk for nonadherence were middle-aged adults with busy schedules. In comparison, older adults who had more routine and less busy lives made fewer errors. If younger adults are making a greater proportion of adherence errors compared to the more cognitively vulnerable population of older adults, then we must examine what noncognitive factors are to blame Indeed, further analysis revealed that it was the level of busyness specifically that seemed most predictive of medication adherence errors.

As Brandimonte and Ferrante (chap 16, this volume) note in their chapter, very little has been published on the social aspects of prospective memory. Most theories within the prospective memory literature tend to be solely cognitive in nature. Based on comparing the prospective memory performance of younger and older adults, Wilson and Park suggest that situational contexts are more determinative of medication adherence performance than cognitive factors. Following Brandimonte and Ferrante, one wonders, therefore, whether the older adults' positive performance in medical adherence may also be produced by a comparatively stronger sociăl motivation (along with different levels of business as suggested by Wilson \& Park). Older adults may have a stronger desire than younger adults to avoid a potential situation in which their memory is seen as unreliable. The stakes may be higher regarding perceived memory functioning for older rather than young adults in terms of both social interaction and social values.

\section{THE IMPORTANCE OF CUES}

The findings from Dismukes (chap. 19, this volume) indicate that interference in the form of distractions may be most to blame for prospective memory failures in aviation. Similarly, within the social cognitive realm, evidence shows that forming strong intentions does not guarantee goal attainment, as there are a number of subsequent implemental problems (distraction, competing goals) that need to be solved successfully (Cohen \& Gollwitzer, chap. 17, this volume; Gollwitzer, 1993). If the environmental context poses obstacles for prospective memory, then, in a sense, one must find a way to control its potentially distracting effects. If we can use the environment to facilitate remembering, then prospective memory will likely be more successful. One way to manipulate our environment to facilitate prospective memory is to establish cues that trigger associated intentions. In that way, we can relieve ourselves of the laborious task of maintaining an intention in mind. Instead, we rely on a prespecified cue that triggers the intention when the cue is encountered.
Dismukes (chap. 19; this volume) asked research participants to choose one of four statements that best described their encoding of intentions. Most participants reported that they did not identify a specific cue to help them trigger an intention. This finding demonstrates that participants were not spontaneously forming cue + intention links. Rather, they seemed to rely on more internal strategies for remembering the intention. By electing not to choose an environmental cue or aid to trigger an intention, participants may have unknowingly made themselves more vulnerable to the environmental context. In their automatic associative module model, McDaniel et al. (1998) proposed that whenever there is sufficient interaction between a prospective cue and an associated memory trace, the memory trace for the intended action is delivered automatically to consciousness. If we accept that proposition, prospective remembering is dependent on the strength of the association between the cue and the associated memory trace. This suggests that the planning and encoding stage of prospective memory is critical for successful performance. Along these lines, Gollwitzer $(1993,1999)$ suggested that one is more likely to achieve a goal when he or she forms a certain type of intention called an implementation intention. As described in Cohen and Gollwitzer (chap. 17 , this volume), an implementation intention is the formation of a plan that specifies how one will respond to a prespecified cue or situation. For example, if I need to remember to turn off the oven after dinner, then I may form an implementation intention that links the desired response with a specific context. If, as Dismukes found, participants are not likely to form cue + intention pairings spontaneously, it may be necessary to have them deliberately form implementation intentions so that they can use the environment to their advantage:

In a clinical population, there is commonly an even greater need to establish strategies that help patients overcome prospective memory deficits. As many scholarly articles on the topic of prospective memory are quick to state, prospective memory is critical to daily functioning (e.g., the need to remember to take medication, turn off a stove). Therefore, patients with brain injuries can suffer severe liabilities in their ability to live independently when their prospective memory functioning is impaired. However, as Thöne-Otto and Walther (chap. 15, this volumé) note in their very thorough review of the literature on clinical assessment and therapy of prospective memory in patients with brain injury, compensatory memory aids often involve external reminders rather than internal strategies. Due to the expense and technological challenges associated with electronic memory aids, there may be a need for more research investigating internal prospective memory strategies.

One study examined the effects of a self-regulatory strategy in a population with brain injuries. Specifically, Lengfelder and Gollwitzer (2001) studied the effects of implementation intentions with frontal lobe patients. Typically, patients with frontal lobe injury display difficulty with tasks that require high levels of conscious control. In contrast, tasks that require more automatic behaviors are not impaired (e.g., Shallice, 1982). Lengfelder and Gollwitzer asked a sample of frontal lobe patients to perform a go/no-go task, as well as a secondary tracking task of varying difficulty. Their results showed that even at a high level of difficulty on the tracking task, implementation intentions improved performance on the go/no-go task. Moreover, the 
effect of implementation intentions was stronger in frontal lobe patients than in a control group of university students. This study showed that using implementation intentions helped participants recruit automatic rather than effortful controlled memory processes, which enhanced their performance.

\section{HOW TO ENHANCE THE POWER OF IMPLEMENTATION INTENTIONS?}

\section{The State of the Superordinate Goal}

Sheeran, Webb, and Gollwitzer (2005, Study 2) found that implementation intention effects are sensitive to the (even subliminal) activation of the superordinate goal. In other words, implementation intentions only affect behavior when the superordinate goal is activated. As a consequence, people who use implementation intentions to avoid forgetting to act on their goals should seek out situational contexts that implicitly or explicitly activate the respective goal. However, implementation intention effects are not only found to be sensitive to goal activation, they also respect the strength of and commitment to the superordinate goal (e.g., Sheeran et al., 2005, Study 1). The more hours college students wanted to engage in independent study, the greater the beneficial effects of respective implementation intentions specifying when, where, and how to study. If implementation intentions fail to benefit goal attainment given that the goal is weak, people have to ensure that goal strength and goal commitment are high. Otherwise they cannot rely on the beneficial effects of forming implementation intentions. It becomes important therefore that people keep up high motivation throughout goal striving. Strong motivation is dependent not only on high perceived desirability, but also on high perceived feasibility of the wanted future event. Accordingly, people have to retain high self-efficacy beliefs throughout the process of goal striving if they want to profit from their if-then plans.

\section{Single Plans or Multiple Plans}

Given the beneficial effects of if-then planning on acting on one's goals, one wonders how many individual if-then plans people should form for any given goal. If the goal at hand can be served in many different situations and various ways (e.g., the goal to do more physical exercise), it seems wise to specify multiple plans that make use of the many suitable opportunities and instrumental goal-directed responses available. Still, there is the question of whether it is better to solely focus on one particular situation and making several plans that link this very situation to various different instrumental goal-directed behaviors, or to focus on just one particular goal-directed behavior and making plans that link it to the various available suitable situations. Or should people go ahead and first list various highly suitable situations and then link each of them to a unique, most fitting, goal-directed behavior? Alternatively, people might first list various instrumental goal-directed behaviors and then select the most appropriate situations for each of these behaviors.
Answers to these questions need to consider the moderators of implementation intention effects as well as the mechanisms on which they are based. Next to high goal strength and goal commitment, a further important moderator is a person's commitment to the formed plan (Gollwitzer \& Sheeran, 2006). Only if a person strongly commits to a formed if-then plan are we to expect beneficial effects on goal attainment. Intuitively it seems easier to commit to plans that specify either one critical situation only (that is then linked to a multitude of goal-directed behaviors) or just one critical goal-directed behavior (that is then linked to a multitude of suitable situations) than to a multitude of if-then links between critical situations and behaviors. However, it is up to empirical research to find decisive answers to this question.

If one considers the mechanisms on which implementation intentions are based; one has to distinguish between the mechanisms that relate to the if part (i.e., identification processes) versus the mechanisms that relate to the then part of implementation intentions (i.e., response initiation processes). The postulated heightened activation of the cues specified in the if part of implementation intentions implies that if-then planning that focuses on one specific cue only and is then linked to multiple goal-directed behaviors is to be preferred over the forming of plans each using a different situational cue (principle of cue competition). The postulated automated initiation of the response specified in the then part of implementation intentions suggests that the formation of plans that link just one goaldirected behavior to a select situational cue should facilitate automation of action initiation and should thus be preferred in comparison to making if-then plans that link various different goal-directed behaviors to one and the same situational cue (principle of response competition)

\section{Individual Differences}

The role of individual differences can also be discussed from various different perspectives. First, there is the straightforward applied question of which people should not bother to make plans, as implementation intentions will fail to have beneficial effects for them. This question should be answered by taking into consideration that implementation intention effects know certain moderators but are based on very simple psychological mechanisms. With respect to moderation, we know that high goal strength and goal commitment are a prerequisite (see earlier) and thus, to give an example, one cannot expect people with strong power and low affiliation motives to benefit from implementation intention formation when trying to meet goals geared at socializing with others. Moreover, as a person's commitment to an if-then plan formed also moderates goal attainment effects (see Gollwitzer \& Sheeran, 2006), certain groups of people may not benefit from forming implementation intentions because they find it aversive to plan out goal striving in advance and thus do not commit to if-then plans (e.g., for individuals high on sociaily prescribed perfectionism, if-then planning was found to arouse negative affect; Powers, Koestner, \& Topciu, 2005)

When one considers the psychological mechanisms on which implementation intention effects are based, it is hard to conceive of a group of individuals who cannot take advantage of these simple mechanisms (i.e., facilitated cue identification 
and automated action initiation). As long as people succeed in specifying suitable cues (i.e., cues that actually arise) in the if part of their implementation intentions, and then link them to instrumental goal-directed responses in the then part that can actually be preformed in the presence of these cues, if-then plans should achieve their beneficial effects. Some groups of individuals may need help with this task (e.g., highly depressed individuals), but once if-then plans with appropriate if and then parts are in place they should succeed in facilitating goal attainment. Indeed, even individuals with chronic problems in action control (e.g., frontal lobe patients, Lengfelder \& Gollwitzer, 2001; schizophrenics and heroin addicts under withdrawal, Brandstätter, Lengfelder, \& Gollwitzer, 2001; children with attention deficit hyperactivity disorder, Gawrilow \& Gollwitzer, in press) showed an increased rate of goal attainment when if-then plans were assigned to them by the experimenter.

Still, there is the issue of whether there are certain individuals who are particularly skilled in forming if-then plans. We have recently started to analyze this question by developing a computer task that allows determining how good a person is in creating strong mental links between anticipated critical cues and goal-directed responses (Grant, Gollwitzer, \& Oettingen, 2006). If one conceives of personality in terms of "intra-individually stable, if ... then ..., situation-behaviour relations" (Mischel \& Shoda, 1995, p. 248), the question of skilful if-then plan formation also refers to the types of situations and responses that are linked. Let us assume that a person has the goal to reduce aggression in relating to others, and he or she also knows about his or her respective situation-behavior profile (i.e., he or she knows what kind of social situations elicit aggressive responses in him or her and how staying calm and collected is possible in other social situations). Given this goal and knowledge, the person can now tailor his or her implementation intentions to those critical, anger-eliciting situations specifying responses that allow him or her to stay calm. Thus, it seems likely that people differ not only in terms of the strength of the if-then links they are able to create but also in terms of coming up with if parts and then parts that take into account their unique chronic situation-behavior profiles, specifying implementation intentions exactly where and how they are needed.

\section{CONCLUSION}

This commentary began by highlighting the fact that most prospective memory laboratory studies do not specify a goal; rather, the majority of studies require participants to encode an arbitrary intention that is removed from daily experience (e.g., "Press the F1 key anytime you see an animal word"). In the absence of a clearly specified goal, it may be that factors determining success or failure in such a task are altogether different from those that influence prospective memory functioning in everyday life. In the social cognitive domain, intentions are formed in the hopes of fulfilling a goal. For example, a person may have the goal of attaining a higher grade-point average (GPA). The student may form an intention to spend four nights a week at the library in the hopes of raising his or her GPA. Thus, intentions are formed in the service of clearly specified goals. Most laboratory prospective memory studies involve instructions that are fairly arbitrary with no clearly specified goal. Future research would benefit by creating goals and intentions that are more in line with an individual's true interests. For example, the goal could be maximizing "points" in an online video game. The intention that would help to attain that goal would be to strike a computer key every time a certain prespecified stimulus appears.

Failures to carry out an intention can result in consequences ranging from burning one's toast to a fatal airplane crash. Relegating our study of prospective memory to controlled laboratory settings has helped us establish some core features of this relatively new area of memory research. However, if we are to continue making theoretical advances, it is vital that we begin to study prospective memory in more varied settings and with more diverse methods. Several chapters in this part present examinations of prospective memory in more diverse settings and they help to underscore the need for continued work in this direction.

\section{REFERENCES}

Brandstätter, V., Lengfelder, A., \& Gollwitzer, P. M. (2001). Implementation intentions and efficient action initiation. Journal of Personality and Social Psychology, 81, 946-960.

Gawrilow, C., \& Gollwitzer, P. M. (in press). Implementation intentions facilitate response inhibitions in children with ADHD. Cognitive Therapy and Research.

Gollwitzer, P. M. (1993). Goal achievement: The role of intentions. European Review of Social Psychology, 4, 141-185.

Gollwitzer, P. M. (1999). Implementation intentions: Strong effects of simple plans. American Psychologist, 54, 493-503.

Gollwitzer, P. M., \& Sheeran, P. (2006). Implementation intentions and goal achievement: A meta-analysis of effects and processes. Advances in Experimental Social Psychology, 38, 69-119.

Grant, H., Gollwitzer, P. M., \& Oetingen, G. (2006). Individual differences in the selfregulation of goal striving by forming implementation intentions. Manuscript submitted for publication.

Lengfelder, A., \& Gollwitzer, P. M. (2001). Reflective and reflexive action control in patients with frontal brain lesions. Neuropsychology, 15, 80-100.

McDaniel, M. A., Robinson-Riegler, B., \& Einstein, G. O. (1998). Prospective remembering: Perceptually driven or conceptually driven processes? Memory \& Congnition, 26, 121-134.

Mischel, W., \& Shoda, Y. (1995). A cognitive-affective system theory of personality: Reconceptualizing situations, dispositions, dynamics, and invariance in personality structure. Psychological Review, 102, 246-268.

Olson, D. R., Astington, J. W., \& Zelazo, P. D. (1999). Introduction: Actions, intentions, and attributions. In P. D. Zelazo, J. W. Astington \& D. R. Olson (Eds.), Developing theories of intention (pp. 1-13). Mahwah, NJ: Erlbaum.

Powers, T. A., Koestner, R., \& Topciu, R. A. (2005). Implementation intentions, perfectionism, and goal progress: Perhaps the road to hell is paved with good intentions. Personality and Social Psychology Bulletin, 31, 902-912.

Sheeran, P., Webb, T. L., \& Gollwitzer, P. M. (2005). The interplay between goal intentions and implementation intentions. Personality and Social Psychology Bulletin, 31, 87-98.

Shallice, T. (1982). Specific impairments of planning. Philosophical Transactions of the Royal Society London, B 298, 199-209. 